rete Berent hatte eine Übersicht über die Lage und die Berufsaussichten der Juristinnen gegeben, die durch Berichte von Assessorinnen, Referendarinnen, Studentinnen und Vertreterinnen anderer Berufsgruppen ergänzt wurden. ${ }^{25}$ Zwei Jahre später findet sich eine Notiz, dass bei der Gründung einer Internationalen Vereinigung von weiblichen Rechtsanwälten in Paris im Juli 1928 als deutsche Vertreterin die Rechtsanwältin Margarete Berent anwesend war. ${ }^{26} 1933$ wandte sich die Schriftführerin des DJV, Lilli Seligsohn, an das preußische Justizministerium, um statistisches Material für Zwecke der Berufsberatung und zur Teilnahme an einer im Frühjahr in Berlin stattfindenden Ausstellung „Die Frau” zu erhalten. Der DJV versandte deshalb einen Fragebogen an alle deutschen Juristinnen, deren Anschriften er neben denen der eigenen Mitglieder ermitteln konnte. ${ }^{27}$

Was mit dem Deutschen Juristinnen-Verein 1933 geschah, ist unklar. Gesichert ist, dass er Ende April 1933 noch bestand $^{28}$, aber im Verlauf der Zeit wohl aufgelöst wurde, um der „Gleichschaltung“ zu entgehen. ${ }^{29}$ Viele seiner Mitglieder mussten emigrieren, einige wurden ermordet wie Cäcilie Holländer, geb. Jacobi, die am 14. November 1941 von Berlin nach Minsk deportiert wurde. Wieviele Mitglieder des DJV nach 1949 noch in Deutschland lebten oder zurückkehrten ist nicht bekannt. Jedenfalls vier aus der Mitgliederliste des DJV von 1919, Dr. Else Heinze-Piorkowski, Dr. Aenne KurowskiSchmitz, Dr. Maria Otto und Alice Pfahl, sind dem 1948 neu gegründeten Juristinnenbund beigetreten.

25 Edelheim, Margarete, Fräulein Richter und Frl. Rechtsanwalt, zwei Jahre Volljuristinnen, in: Vossische Zeitung, 20.6.1926, o.S.

26 Meldung im Berliner Tageblatt vom 22.7.1928, o.S., in: BA R 3001/4182, S. 75 .

27 Deutscher Juristinnenverein, Berlin, den 2.2.1933, an das Preußische Justizministerium., GStA Rep. 84 a, Fiche 12989, Bl. 219.

28 Dankesschreiben des Deutschen Juristinnen-Vereins an Margarete Berent am 29.4.1933 für ihre Arbeit. Margarete Berent Collection, LBI NYC.

29 So Zahn-Harnack, Agnes, Geschichte des deutschen Akademikerinnenbundes, 1926-1933, in: dies., Schriften und Reden 1914-1950, Tübingen 1964, S. 1-8 (7); vgl. auch Huerkamp, Bildungsbürgerinnen, S. 287.

\title{
Juristinnen 1933 bis 1949 - unter besonderer Berücksichtigung der Situation jüdischer Frauen
}

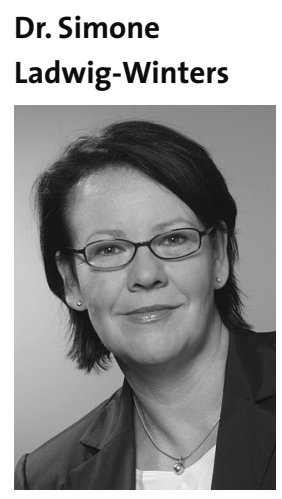

Historikerin, Berlin
Margarete Berent, 1887 in Berlin geboren, wartete lange darauf, die formale Qualifikation einer Volljuristin zu erlangen. ${ }^{1}$ Trotz Studiums und einer Dissertation $^{2}$, die 1913 so viel Anerkennung gefunden hatte, dass Otto von Gierke sie in seiner angesehenen Schriftenreihe veröffentlichte, wurde ihr die Zulassung zum Staatsexamen verweigert. Sie übernahm juristische Hilfsarbeiter- und Wohlfahrtsberaterstellen, war während des Krieges Dezernentin im juristischen Büro der AEG, bis sie 1917 erst beim Zweckverband Groß-Berlin, dann in der Stadtverwaltung Groß-Berlins angestellt wurde. 1919, sobald sich die Möglichkeit in Preußen bot, legte sie dann endlich ihr erstes Staatsexamen ab. Doch der Vorbereitungsdienst blieb ihr vorerst weiterhin verschlossen. Sobald sich hier die Möglichkeit bot, 1922, meldete sie sich dazu und bestand 1925 die große Staatsprüfung. Im gleichen Jahr ließ sie sich im Alter von 38 Jahren als Rechtsanwältin in Berlin nieder.

Margarete Berent ist ein Beispiel dafür, dass sich mit der Zulassung von Frauen zum Staatsexamen zu Beginn der Weimarer Zeit ganz neue Perspektiven eröffneten. Nun konnten Frauen dauerhaft als Juristinnen arbeiten. Damit erweiterte sich das Spektrum der möglichen Berufe, und sie konnten sich nach der Assessorprüfung als Anwältinnen niederlassen oder das Amt einer Richterin oder gar Staatsanwältin übernehmen.
Eine der wenigen, denen es gelang, in den Staatsdienst zu kommen, war Marie Munk (geb. 1885). ${ }^{3}$ Auch sie hatte Umwege machen müssen, um Volljuristin zu werden. Dass sie eine sehr gute Juristin war, erkannten auch die öffentlichen Stellen. Nach einer fünfjährigen Tätigkeit als Anwältin bewarb sie sich für das Amt einer Richterin und wurde tatsächlich 1930 zur ersten Landgerichtsrätin in Preußen ernannt.

Marie Munk kam, wie Margarete Berent oder auch Hanna Katz, aus einer jüdischen Familie. Gerade die jüdischen Familien waren aufgeschlossener, Töchter den Weg in den so männlich dominierten Bereich beschreiten zu lassen. Die Nähe zum Recht war durch die Religion, in der schon immer über Streitfragen diskutiert wurde, vertraut.

1 Zum Leben Berents, die auch eine der Mitbegründerinnen des Juristinnen-Vereins e.V. gewesen ist, s. Ladwig-Winters, Simone: Anwalt ohne Recht - Das Schicksal jüdischer Rechtsanwälte in Berlin nach 1933. 2. Aufl. Berlin 2007; S. 120; Röwekamp, Marion: Juristinnen Lexikon zu Leben und Werk, hrsg. vom Deutschen Juristinnenbund e.V., Baden-Baden 2005, S. 36-40.

2 Berent, Margarete: Die Zugewinnstgemeinschaft der Ehegatten. Breslau 1915, in der Reihe „Untersuchungen zur deutschen Staats- und Rechtsgeschichte, Heft 123; diese Dissertation fand dann viele Jahre später, 1959, bei der Eherechtsreform der Bundesrepublik Deutschland Berücksichtigung.

3 Personalakte, s. BArch R 3001, 69.257; Röwekamp (2005), S. 275-279. 
Für Angehörige einer Minderheit war es zudem sinnvoll und notwendig, die rechtlichen Gegebenheiten der Mehrheitsgesellschaft zu kennen, schon um die eigene Position zu sichern und Konflikte zu vermeiden. Deshalb war der Anteil von Juden unter den Juristen deutlich höher, als es ihrem Bevölkerungsanteil entsprach. Jüdische Frauen, die sich in der Weimarer Republik als Juristinnen etablierten, gehörten damit im doppelten Sinne einer Minderheit an.

\section{Der Bruch 1933}

Die Machtübernahme der Nationalsozialisten 1933 bedeutete einen massiven Bruch. Die ideologischen Ansätze wurden schnell in Politik umgeformt. Das betraf nicht nur die Rassenoder Wirtschaftspolitik, sondern auch die Familienpolitik und die Rolle der Frau innerhalb der Gesellschaft.

\section{Antisemitismus als staatliches Ziel}

Mit rassistischem Impetus wurden innerhalb von nur drei Monaten das Gesetz zur Wiederherstellung des Berufsbeamtentums (vom 7.4.1933, RGBl. I S. 175-177) sowie das Gesetz über die Zulassung zur Rechtsanwaltschaft (vom 7.4.1933, RGBl. I S. 188) erlassen.

Diesen Regelungen waren terroristische Angriffe auf bekannte Gegner der Nationalsozialisten und exponierte Persönlichkeiten vorangegangen. In Berlin war der junge Anwalt Hans Litten, der Hitler 1931 bei einem Prozess in Bedrängnis gebracht hatte, in so genannte Schutzhaft genommen worden, in Frankfurt der bekannte Arbeitsrechtler und Rechtssoziologe Prof. Dr. Hugo Sinzheimer. Erste Todesfälle waren zu verzeichnen: Dr. Max Plaut in Kassel und Günter Joachim in Berlin wurden erschlagen. Ende März kam es zu verschiedenen Übergriffen auf Gerichte. In Köln musste sich Rechtsanwältin Ruth Bodenheimer am 31. März 1933 vor den aufgeputschten SA-Truppen in Sicherheit bringen. ${ }^{4}$ Ansonsten sind keine weiteren Frauen in dieser Phase unmittelbar bedroht worden.

In Preußen erließ Hanns Kerrl den so genannten Kerrl'schen Erlass, der ein Hausverbot für jüdische Richter und Richterinnen, Staatsanwälte [nach bisherigem Wissen gab es nur männliche Staatsanwälte], Rechtsanwälte und Rechtsanwältinnen bedeutete. In Sachsen und Bayern gab es ähnliche Erlasse. In der Folge wurde die Ausgrenzung auf der Grundlage der beiden oben benannten Gesetze systematisiert.

Auf diesem Wege verloren Menschen jüdischer Religion oder Herkunft ihren Beruf. Die Ausnahmeregelungen, die gewährt wurden, konnten allein von Männern in Anspruch genommen werden. Denn sie wurden nur denjenigen zugestanden, die vor 1914 in den Dienst aufgenommen oder als Anwalt zugelassen worden waren oder sich als „Frontkämpfer“ bewährt hatten. Das konnte für Frauen nicht gelten. Da sie erst nach Ende des Kaiserreichs die Möglichkeit erhalten hatten, die Staatsexamina abzulegen, konnten sie nicht vor 1914 in ihre Stellung gelangt sein, und während des Weltkriegs hatten sie ebenfalls nicht mit der Waffe dienen können. So wurden jüdische Juristinnen 1933 aus rassistischen Gründen mit Berufsverbot belegt.

\section{Rassistische Ausgrenzung der jüdischen Beamtinnen}

Von den neun ${ }^{5}$ in Preußen eingesetzten Richterinnen oder ständigen (richterlichen) Hilfsarbeiterinnen waren vier jüdischer Herkunft. Drei von ihnen wurden auf der Grundlage von $\mathbb{3}$ BBG ,in den Ruhestand versetzt““. ${ }^{6}$ Marie Munk, die evangelischer Religion, aber nach nationalsozialistischer Definition Jüdin war, gehörte zu den Ersten, die den neuen Bestimmungen zum Opfer fielen. Wie auch die anderen stand sie wirtschaftlich vor dem Nichts, denn die Bewilligung eines Ruhegeldes hätte eine zehnjährige Dienstzeit vorausgesetzt. Eine alternative Betätigung als Anwältin war durch das Anwaltsgesetz ebenfalls ausgeschlossen. Wie ihr erging es den Gerichtsassessorinnen, die jüdischer Religion oder Herkunft waren. Das Reichsbeamtengesetz vom 30. Juni 1933 hatte hier noch weiter eingegrenzt, dass keine „nichtarischen“ oder mit „Nichtariern“ verheirateten Personen berufen werden durften. ${ }^{7}$ Eine von ihnen war die junge Elsbeth von Ameln. Sie hatte zwar noch 1934, kurz vor ihrem 29. Geburtstag, ihr zweites Staatsexamen ablegen können, doch bot sich auch ihr keine Chance für eine weitere Berufsausübung. ${ }^{8}$

\section{Rassistische Ausgrenzung der jüdischen Rechtsanwältinnen}

Für die niedergelassenen Anwältinnen war die Situation nicht anders. 1933 sahen sich in Berlin 19 Anwältinnen mit der rassistischen Ausgrenzung konfrontiert. ${ }^{9}$ Keine wollte ihren hart errungenen Beruf aufgeben, doch umgehend wurden Vertretungsverbote ausgesprochen. Sorgfältig prüfte die Justizverwaltung die Anträge auf weitere Zulassung, die bei den jeweiligen Kammern eingereicht worden waren, um schließlich (zumeist) zum Sommer 1933 unbefristete Berufsverbote zu erlassen. Dieses Verfahren wurde im gesamten Deutschen Reich praktiziert, im Norden wie im Süden; in München traf es zum Beispiel drei Anwältinnen. ${ }^{10}$

Einige jüdische Anwältinnen, deren Ehemänner noch weiter praktizieren durften, arbeiteten dann in der Kanzlei des Mannes mit. Die Ausgrenzung wurde noch ausgeweitet: so wurden auch nichtjüdische Frauen, die in einer so genannten Mischehe lebten, durch die gesetzliche Regelung vom 30. Juni 1933 ( $\$ 1$ a Abs. 3 Reichsbeamtengesetz) erfasst und konnten auf Grund ihrer Ehe mit einem „nicht arischen“ Mann entlassen werden.

4 Luig, Klaus: ...weil er nicht arischer Abstammung ist. Jüdische Juristen in Köln während der NS-Zeit. Köln 2004, S. 121.

5 Geheimes Staatsarchiv, Rep. 84a, Nr. 2945, Bl. 97 ff., Verzeichnis der weiblichen Richterkräfte, Stand: 12.8.1933; hier sind sechs Frauen aufgeführt, darunter Erna Haßlacher. Zu diesem Zeitpunkt war aber bereits gegen einige ein Berufsverbot nach $\S 3$ BBG ausgesprochen worden.

6 Bergemann, Hans/Ladwig-Winters, Simone: Richter und Staatsanwälte jüdischer Herkunft in Preußen im Nationalsozialismus. Eine Dokumentation. Köln 2004, S. 55/56. Marie Munk, Hedwig Brann und Else Guttmann.

7 \$ 1 Abs. 3 Reichsbeamtengesetz in der Fassung vom 30.6.1933.

8 Luig (2004), S. 105/106.

9 Ladwig-Winters (2007), S. $58 \mathrm{ff}$.

10 Weber, Reinhard: Das Schicksal der jüdischen Rechtsanwälte in Bayern nach 1933. München 2006, S. 78. 
Ganz offiziell gab es nach 1933 in ganz Deutschland keine jüdische Juristin mehr, die ihren Beruf ausüben durfte - bis auf eine Ausnahme: Hanna Katz. ${ }^{11}$ Hanna Katz hatte 1925 ihr erstes Staatsexamen abgelegt und sich nach dem Assessorexamen als Anwältin in Berlin niedergelassen. Mit der zugleich erworbenen Kompetenz einer vereidigten Dolmetscherin und einem großen Interesse für Wirtschaftsrecht war sie Mitglied der International Law Association geworden und hatte dort im Trade Mark Committee das Amt der Schriftführerin übernommen. Durch das Berufsverbot hätte Hanna Katz ihren Posten in der Internationalen Juristenvereinigung verloren. Da kein deutscher Vertreter, sondern ein englischer nachgerückt wäre, zogen es die deutschen Behörden vor, sie weiterhin als Ver-

\section{Neben der rassistischen Ausgrenzung war die politische Unterdrückung und Verfolgung der Gegnerinnen des Nationalsozialismus ein zentrales Ziel.}

treterin zu entsenden, deshalb wurde gegen sie kein Berufsverbot verhängt. Sie blieb sogar von dem allgemeinen Berufsverbot für jüdische Anwälte zum November 1938 verschont und wurde als einzige „Konsulentin“, als jüdische Rechtsvertreterin von Juden bzw. denen, die dies nach nationalsozialistischer Definition waren, zugelassen. Gerade noch rechtzeitig gelang es ihr 1941, aus Deutschland in die USA zu fliehen.

\section{Weiterer Lebensweg}

Wie Hanna Katz gelang es auch Margarete Berent und Marie Munk, in die USA zu emigrieren, was angesichts der beschränkten Aufnahmezahlen ein äußerst schwieriges Unterfangen war. Sie mussten noch einmal von vorn anfangen, da kein staatlicher Abschluss anerkannt wurde. Sie alle studierten erneut und ließen sich dann als Anwältinnen in New York nieder. Margarete Berent war zu diesem Zeitpunkt bereits 63 Jahre alt. Doch die Einkünfte waren gering, zu gering, um ein Auskommen zu haben. Deshalb konnte sie sich glücklich schätzen, dass sie eine Stellung in der Rechtsabteilung der Stadt New York fand, die sie bis kurz vor ihrem Tode 1965 innehatte.

Andere, die nicht aus Deutschland hatten fliehen können, wurden ermordet. So die Münchener Anwältin Elisabeth Kohn (1902-1941), die in Kovno (dem heutigen Kaunas) gemeinsam mit ihrer Mutter und ihrer Schwester erschossen wurde. ${ }^{12}$ Mindestens vier weitere Juristinnen kamen ebenfalls im Zuge der nationalsozialistischen Verfolgung ums Leben. ${ }^{13}$
Einige wenige überlebten in Deutschland, weil sie „untertauchten“, d.h. in die Illegalität gingen. $\mathrm{Zu}$ ihnen gehörte Anita Eisner, Anwältin aus Berlin, die sich nach dem Berufsverbot 1933 noch eine gewisse Zeit mit Haus- und Vermögensverwaltertätigkeiten durchschlagen konnte; angesichts der sich immer mehr zuspitzenden Verfolgung tauchte sie unter und wurde vollständig von der Unterstützung von Freunden und Bekannten abhängig.

\section{Politische Ausgrenzung}

Neben der rassistischen Ausgrenzung war die politische Unterdrückung und Verfolgung der Gegnerinnen des Nationalsozialismus ein zentrales Ziel der Politik nach 1933. Hatten sich in der Weimarer Republik Strafverteidigerinnen und Strafverteidiger in der Roten Hilfe Deutschlands als Schutz- und Solidaritätsorganisation für Arbeiter in politischen Prozessen zusammengeschlossen, wurden sie nun selbst Opfer der politischen Verfolgung. ${ }^{14}$ Der Roten Hilfe gehörten Hilde Benjamin geb. Lange ${ }^{15}$, Dr. Nora Block ${ }^{16}$, Dr. Elfriede Cohnen ${ }^{17}$, die bereits erwähnte Elisabeth Kohn sowie Hilde Kirchheimer, spätere Neumann ${ }^{18}$ an. Außer bei Hilde Benjamin

11 Zum Leben Katz', s. Ladwig-Winters (2007), S. 192/93; Röwekamp (2005), S. 168-172.

12 Schneider, Heinz-Jürgen/Schwarz, Erika/Schwarz, Josef: Die Rechtsanwälte der Roten Hilfe Deutschlands. Politische Strafverteidiger in der Weimarer Republik. Bonn 2002, S. 182; Röwekamp (2005), S. 193-95; Weber (2006), S. 241.

13 Bekannt ist das Schicksal von Clara Israel (1876-1942), von 1929 bis 1933 Magistratsrat, Suizid angesichts der bevorstehenden Deportation, s. Göppinger, Horst: Juristen jüdischer Abstammung im ,Dritten Reich ‘ - Entrechtung und Verfolgung. 2. Aufl. München 1990, S. 233. Ella Kessler-Reis (1899-1944), Anwältin in Stuttgart, die in Auschwitz ermordet wurde, s. Bundesarchiv Koblenz (Hrsg.): Gedenkbuch - Opfer der Verfolgung der Juden unter der nationalsozialistischen Gewaltherrschaft in Deutschland 1933-1945, 2. erw. Aufl. Berlin 2006, S. 1714; Göppinger (1990), S. 249/259; Stuttgarter StolpersteinInitiative: www.stolpersteine-stuttgart.de (2008). Guttmann geb. Samulon (1898-Deportation nach Auschwitz 1944), Richterin aus Berlin, s. Bergemann/Ladwig-Winters (2004), S. 192; Göppinger (1990), S. 258. Dr. Erika Sienauer (1898-Deportation nach Auschwitz), Rechtsanwältin aus Freiburg, s. Göppinger (1990), S. 26o. Nicht für alle Juristinnen ist ihr weiterer Lebensweg bekannt. Zu einigen Anwältinnen wurde im Rahmen der Wanderausstellung „Anwalt ohne Recht“, die von der Bundesrechtsanwaltskammer und dem Deutschen Juristentag seit 1990 veranstaltet wird, Näheres über ihr Schicksal ermittelt, s. Bundesrechtsanwaltskammer (Hrsg.): Anwalt ohne Recht. Schicksale jüdischer Rechtsanwälte in Deutschland nach 1933. Katalog zur gleichnamigen Ausstellung. Berlin 2007.

14 Schneider u.a. (2002).

15 Schneider u.a. (2002), S. 86; Röwekamp (2005), S. 28-33.

16 Schneider u.a. (2002), S. 91; Röwekamp (2005), S. 294298, hier Nora Platiel geb. Block.

17 Schneider u.a. (2002), S. 105.

18 Zu Hilde Neumann geb. Rosenfeld gesch. Kirchheimer, s. Ladwig-Winters (2007), S. 195; Schneider u.a. (2002), S. 224; Röwekamp (2005), S. 280/81. 
fiel bei allen Frauen die politische mit der rassistischen Verfolgung zusammen, so dass sie schon aus diesem Grund ihre Zulassungen als Anwältinnen verloren. Gegen Benjamin wurde 1933 ein Vertretungsverbot erlassen; sie gab in der Folge ihre Kanzlei auf, zumal sie mit weiteren Schritten rechnen konnte, denn auch ihr Mann Georg Benjamin war politisch aktiv und zudem noch Jude.

Im Zuge der Gleichschaltung wurden alle selbstständigen Vereine und Organisationen zwangsweise aufgelöst oder vorrangig mit Nationalsozialisten besetzt. Um diesen Maßnahmen zu entgehen, löste sich auch der Deutsche Juristinnen-Verein im April 1933 auf. Die gewählten Vertreter der selbstständigen Kammern als Vertretungen der Anwaltschaft wurden in dieser Zeit hinausgedrängt und durch Anhänger der NSDAP ersetzt. Auf diese Weise entfiel jegliche Form der unabhängigen Selbstorganisation und politischen Vertretung.

\section{Diskriminierung von Frauen allgemein}

Da die nationalsozialistische Politik, anders als bei der jüdischen Minderheit oder den politischen Gegnern, auf die gesellschaftliche Mitwirkung von Frauen angewiesen, ihre verstärkte politische Teilhabe jedoch unerwünscht war, wurde beständig das Bild der Frau den aktuell geltenden Vorstellungen angepasst. Frauen verloren umgehend das passive Wahlrecht. Dieses Vorgehen lässt sich auch auf die Rechtspflege übertragen. Die Blockierung ihrer Laufbahnen erfolgte verdeckt; umgehend wurden Neubesetzungen beschränkt. So passten Frauen auf einmal nicht mehr in das politisch dominierte System der Ausbildung, das über Referendarausbildungslager vermittelt wurde, wie z.B. das gleich 1933 in Jüterbog errichtete. Hier wurde ein körperbetontes, einseitig männlich orientiertes Bild des „neuen“ Juristen entwickelt, in dem Frauen keinen Platz hatten.

Zudem konnten Frauen nur noch eine Planstelle erlangen, wenn sie mindestens 35 Jahre alt waren. ${ }^{19}$ Über die Bekämpfung des „Doppelverdienertums“ wurde ebenfalls gegen bereits eingestellte Frauen vorgegangen: Wenn sie verbeamtet und verheiratet waren, liefen sie Gefahr, ihren Beruf zu verlieren. Grundlage dafür bildete das Deutsche Beamtengesetz vom 26. Januar 1937 (RGBl. I, S. 39 ff.). ${ }^{20}$

Diejenigen, die eine feste Stelle hatten und aus keinem Grund aus dem Beruf gedrängt werden konnten, mussten sich oftmals Versetzungen gefallen lassen, sodass „sich Frauen praktisch nur in der freiwilligen Gerichtsbarkeit" noch fanden. ${ }^{21}$

Selbst als nach Kriegsbeginn der Personalmangel immer gravierender wurde, weil viele männliche Juristen beim Militär waren, setzte man Frauen auf Planstellen lediglich in der Verwaltung ein. ${ }^{22}$ Inner- halb der Anwaltschaft gab es diese Beschränkungen für zugelassene Anwältinnen nicht. Juristinnen wie Magdalene Schoch ${ }^{23}$, die einzige habilitierte Frau (Habilitation 1932) -, die in die Wissenschaft gegangen waren, sahen keine Chancen, unter diesen politischen Verhältnissen frei zu lehren und zu forschen. Magdalene Schoch ging 1937 in die Emigration und blieb dauerhaft in den USA.

\section{Selbst als nach Kriegsbeginn der Personalmangel immer} gravierender wurde, weil viele männliche Juristen beim Militär waren, setzte man Frauen auf Planstellen lediglich in der Verwaltung ein. Innerhalb der Anwaltschaft gab es diese Beschränkungen für zugelassene Anwältinnen nicht.

\section{Nach 1945}

Einzig der Vorteil der geringeren Verstrickung mit dem politischen System hatte sich aus der offenen und der versteckten Diskriminierung ergeben. Weil sich viele beruflich nicht integrieren konnten, wurden sie auch nicht im gleichen Maße wie männliche Juristen an dem strukturellen Unrechtssystem beteiligt. Doch bedurfte es anschließend eines erheblichen Zeitraums, bis sich wieder verstärkt Frauen in der Jurisprudenz betätigten.

Für diejenigen, die in Deutschland die rassistische Verfolgung überlebt hatten oder aus dem Exil zurückgekehrt waren, bedeutete das, dass sie als „unbelastet“ eingestuft werden konnten. Sie boten die Gewähr, beim Aufbau der Justiz nicht die nationalsozialistischen Ideen weiter zu perpetuieren, deshalb sollten sie (wie auch jüdische Anwälte) in der Gerichtsbarkeit eingesetzt werden. Dafür mussten sie in den Zonen der westlichen Alliierten ein Entnazifizierungsverfahren durchlaufen. Im Fall von Anita Eisner lässt sich nachzeichnen, dass sie die Verpflichtung, zum Richteramt herangezogen zu werden, ablehnte und es vorzog, wieder als Anwältin zugelassen zu werden. ${ }^{24}$

19 §1 a Abs. 3 Reichsbeamtengesetz (vom 30.6.1933, RGBI. IS. 433).

20 Flügge, Sibylla: Der lange Weg in die Gerichte. Von der Männlichkeit des Staates und vom Ende holder Weiblichkeit. Streit 4/84, S. 149-153, hier: 152.

21 Deutscher Juristinnenbund e.V. (Hrsg.): Juristinnen in Deutschland. Die Zeit von 1900 bis 1998. 3. neu bearb. Aufl. Baden-Baden 1998, S. 25.

22 Deutscher Juristinnenbund (1998), S. 27.

23 Nicolaysen, Rainer: Für Recht und Gerechtigkeit. Über das couragierte Leben der Juristin Magdalene Schoch (1897-1987), Zeitschrift des Vereins für Hamburgische Geschichte 92 (2006), S. 113-143; Röwekamp (2005), S. 368-372.

24 Anita Eisner starb aber kurz danach, auf Grund der langen Verfolgung, im Alter von 50 Jahren, s. Ladwig-Winters (2007), S. 144/45. 
Als dagegen Erna Proskauer aus dem Exil zurückgekehrte und sich nun erneut um ein Richteramt bewarb, wurde ihr dies in West-Berlin verweigert. Sie hatte 1933 als Assessorin auf ein Richteramt gehofft, auch 20 Jahre später fand sich keine Stelle für sie. ${ }^{25}$ Sie ließ sich daher als Anwältin nieder. Anders dagegen Käthe Manasse-Loewy. Sie war 1933 bereits Anwältin in Berlin gewesen, kehrte 1949 nach dem Exil, ebenfalls in Palästina und dann in Israel, nach Deutschland zurück, als sie ihrem Mann folgte. 1953 wurde sie schließlich zur Richterin ernannt, gegen den Widerstand des Präsidenten des Hanseatischen Oberlandesgerichts, der sich dagegen aussprach, dass sich eine mit einem niedergelassenen Anwalt verheiratete Juristin als Richterin betätigte. $^{26}$

Dass die politische Orientierung vor 1933 ausschlaggebend dafür war, wo man sich nach Kriegsende niederließ, ist kaum überraschend. Kommunistinnen, die entweder schon vor 1933 politisch aktiv gewesen oder dann durch den Widerstand gegen den Nationalsozialismus zu ihrer Überzeugung gelangt waren, waren oftmals in die Sowjetunion ins Exil gegangen. Sie kehrten entsprechend in die sowjetische Zone zurück und engagierten sich für den Aufbau eines sozialistischen Staates auf deutschem Boden. Exemplarisch lassen sich hier Hilde Neumann gesch. Kirchheimer ${ }^{27}$ und Hilde Benjamin nennen. Benjamins Mann Georg war 1942 im Konzentrationslager Mauthausen unter ungeklärten Umständen ums Leben gekommen.

\section{Literatur}

Bergemann, Hans/Ladwig-Winters, Simone: Richter und Staatsanwälte jüdischer Herkunft in Preußen im Nationalsozialismus. Eine Dokumentation. Köln 2004.

Brentzel, Marianne: Die Machtfrau. Hilde Benjamin 1902-1989. Berlin 1997.

Bundesrechtsanwaltskammer (Hg.): Anwalt ohne Recht. Schicksale jüdischer Rechtsanwälte in Deutschland nach 1933. Katalog zur gleichnamigen Ausstellung. Berlin 2007.

Bundesarchiv Koblenz (Hg.): Gedenkbuch - Opfer der Verfolgung der Juden unter der nationalsozialistischen Gewaltherrschaft in Deutschland 1933-1945, 2. erw. Aufl. Berlin 2006.

Deutscher Juristinnenbund e.V. (Hg.): Juristinnen in Deutschland. Die Zeit von 1900 bis 1998. 3. neu bearb. Aufl. Baden-Baden 1998.

Feth, Andrea: Hilde Benjamin. Eine Biographie. Berlin 1997.

Göppinger, Horst: Juristen jüdischer Abstammung im `Dritten Reich ‘Entrechtung und Verfolgung. 2. Aufl. München 1990.

Ladwig-Winters, Simone: Anwalt ohne Recht - Das Schicksal jüdischer Rechtsanwälte in Berlin nach 1933. 2. Aufl. Berlin 2007.

Luig, Klaus: ...weil er nicht arischer Abstammung ist. Jüdische Juristen in Köln während der NS-Zeit. Köln 2004.

Nicolaysen, Rainer: Für Recht und Gerechtigkeit. Über das couragierte Leben der Juristin Magdalene Schoch (1897-1987), Zeitschrift des Vereins für Hamburgische Geschichte 92 (2006).

Proskauer, Erna:Wege und Umwege. Erinnerungen einer Berliner Rechtsanwältin. Frankfurt a.M. 1996.

Röwekamp, Marion: Juristinnen - Lexikon zu Leben und Werk, hg. vom Deutschen Juristinnenbund e.V., Baden-Baden 2005.

Schneider, Heinz-Jürgen/Schwarz, Erika/Schwarz, Josef: Die Rechtsanwälte der Roten Hilfe Deutschlands. Politische Strafverteidiger in der Weimarer Republik. Bonn 2002.

Weber, Reinhard: Das Schicksal der jüdischen Rechtsanwälte in Bayern nach 1933. München 2006.
Seine Witwe siedelte 1948, nachdem sie lange Zeit im Westteil Berlins gelebt hatte, in den sowjetisch besetzten Teil über. Sie war gleich im Mai 1945 zur Richterin ernannt worden und baute dann an verantwortlicher Stelle die Justiz in der Sowjetischen Besatzungszone sowie der DDR mit auf. ${ }^{28}$

Wie bereits die Zulassung zu den juristischen Berufen nach 1919 zeigte, waren rund zehn Jahre nötig, bis sich nach der Ausbildung eine nennenswerte Gruppe von Frauen herausbildete, die sich als Juristinnen betätigen konnten. In der Zeit des Nationalsozialismus war die berufliche Tätigkeit von Frauen aus verschiedenen Gründen eingeschränkt worden, entsprechend fehlte nach Kriegsende fast eine ganze Generation von Juristinnen.

25 Proskauer, Erna: Wege und Umwege. Erinnerungen einer Berliner Rechtsanwältin. Frankfurt a.M. 1996; s. auch Ladwig-Winters: Die Nazis versperrten ihr die Laufbahn als Juristin. Nachruf auf Erna Proskauer, Frankfurter Allgemeine Zeitung vom 1.2.2001.

26 Röwekamp (2005), S. 237.

27 Ladwig-Winters (2007), S. 195; Röwekamp (2005), S. 288/81.

28 Nach Gründung der DDR war sie wieder Richterin und in dieser Funktion mitverantwortlich für verschiedene Todesurteile, und bekleidete von 1953 bis 1967 das Amt der Justizministerin der DDR: s. Röwekamp (2005), S. 28-33; Brentzel, Marianne: Die Machtfrau. Hilde Benjamin 1902-1989. Berlin 1997; Feth, Andrea: Hilde Benjamin. Eine Biographie. Berlin 1997.

\section{Dokumente/Archivalien/Artikel}

Berent, Margarete: Die Zugewinnstgemeinschaft der Ehegatten. Breslau 1915, in der Reihe „Untersuchungen zur deutschen Staats- und Rechtsgeschichte", Heft 123.

BArch R 3001, 69.257, Bestände des Reichsministeriums der Justiz, Personalakte.

Flügge, Sibylla: Der lange Weg in die Gerichte. Von der Männlichkeit des Staates und vom Ende holder Weiblichkeit. Streit 4/84, S. 149-153.

Geheimes Staatsarchiv, Rep. 84a, Nr. 2945, Bestände des Preußischen Justiministeriums.

Ladwig-Winters, Simone: Die Nazis versperrten ihr die Laufbahn als Juristin. Nachruf auf Erna Proskauer, Frankfurter Allgemeine Zeitung vom 1.2.2001.

\section{Internetquellen}

www.stolpersteine-stuttgart.de (2008) - Stuttgarter Stolperstein-Initiative. 